\title{
Single-Port-Access Laparoscopic Radical Trachelectomy in Early Stage Cervical Cancer with Successful Pregnancy Outcome: A Case Report
}

\author{
Athithan Rattanaburi, M.D. ${ }^{1}$, Yuyeon Jung, M.D. ${ }^{2}$, Jung Hwan Ahn, M.D. ${ }^{2}$, \\ Keun Ho Lee, M.D., Ph.D. ${ }^{2}$, Ji Geun Yoo, M.D. ${ }^{3}$
}

'Division of Gynecologic Oncology, Department of Obstetrics and Gynecology, Faculty of Medicine, Prince of Songkla University, Hat Yai, Songkhla 90110, Thailand.

${ }^{2}$ Department of Obstetrics and Gynecology, Seoul St. Mary's Hospital, College of Medicine, The Catholic University of Korea, Seoul 06591, Republic of Korea.

${ }^{3}$ Department of Obstetrics and Gynecology, St. Vincent Hospital, Suwon, Gyeonggi 16247, Republic of Korea.

Received 28 February 2020 • Revised 19 April 2020 • Accepted 21 April 2020 • Published online 21 July 2020

\begin{abstract}
:
A 31-year-old woman, gravida 0, was found to have atypical glandular cells on her cytology cervical smear. A colposcopy-directed biopsy revealed adenocarcinoma. Without a definite endocervical mass extension on a magnetic resonance imaging, a single-port-access laparoscopic radical trachelectomy (SPA-RT) was performed. The final pathology was adenocarcinoma with a free section margin. She resumed her normal menstrual cycle a month after the operation and a few months later became naturally pregnant, delivering a viable infant at 34 weeks of gestation by cesarean section. At the time of this writing, 64 months after the SPA-RT, she is still free from recurrent disease.
\end{abstract}

Keywords: case report, outcome, radical trachelectomy, single-port surgery

\section{Contact: Keun Ho Lee, M.D., Ph.D.}

Department of Obstetrics and Gynecology, Seoul St. Mary's Hospital, College of Medicine,

The Catholic University of Korea, Seoul 06591, Republic of Korea.

E-mail: hohoho@ catholic.ac.kr

. Hosting by Prince of Songkla University. All rights reserved.

This is an open access article under the CC BY-NC-ND license

(http://www.jhsmr.org/index.php/jhsmr/about/editorialPolicies\#openAccessPolicy). 


\section{Introduction}

Radical trachelectomy is considered as a standard surgical treatment modality for women diagnosed with early stage cervical cancer wishing to preserve their fertility. Minimally invasive approaches to radical trachelectomy have reported some benefits over laparotomy such as less blood loss and a shorter hospital stay. ${ }^{1}$ However, maintaining good rates of post-operative fertility and obstetric outcomes might be challenging aspects of this procedure. Single-port-access laparoscopic surgery is feasible and has some benefits compared with multiport-access laparoscopic surgery for many gynecologic surgical procedures ${ }^{2,3}$, however to date there are no studies which have examined single-port-access radical trachelectomy (SPA-RT) for feasibility, optimal surgical technique, or oncological and obstetrical outcomes. Herein we describe a case in which SPA-RT was successfully conducted in the interests of surgical skills education. The patient gave written informed consent for the use of her story.

\section{Case report}

A 31-year-old woman, gravida 0 , came for asymptomatic cervical cancer screening in August 2014. A liquid-based cytology test revealed atypical glandular cells, and human papilloma virus (HPV) genotyping via real-time polymerase chain reaction was positive for the HPV 18 genotype. A physical examination revealed a nodular lesion size 1.5 centimeters $(\mathrm{cm})$ at the cervix (Figure 1A). Vaginal fornices and parametrium were normal. Colposcopy revealed a glandular proliferative area $2 \times 1.5 \mathrm{~cm}$ on the cervix without atypical vessels (Figure 1B). A cervical biopsy showed a well-differentiated adenocarcinoma of the cervix. Magnetic resonance

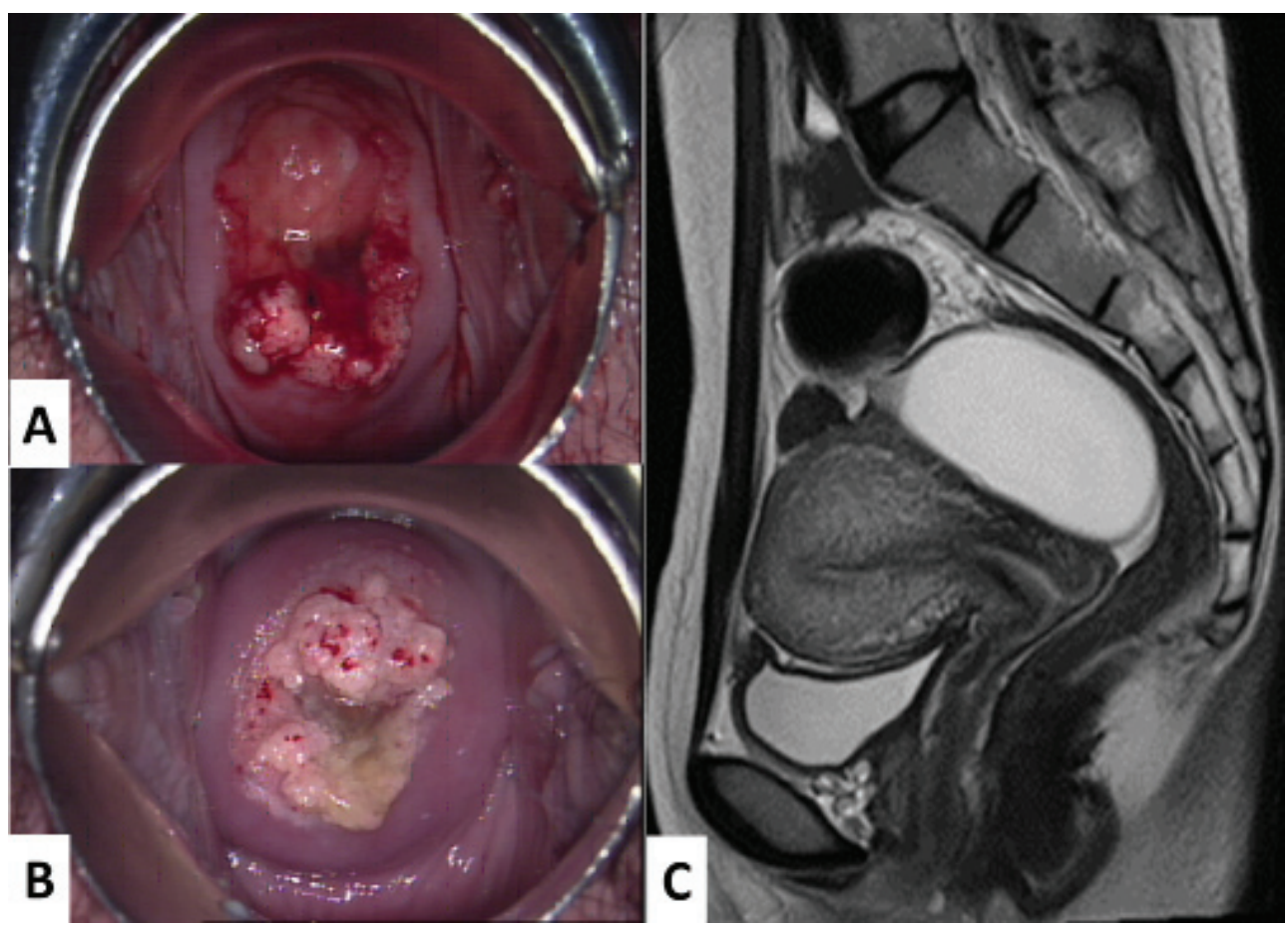

Figure $1 \mathrm{~A}$ : Cervical lesion on physical examination B: Colposcopy revealed a glandular proliferative area $2 \times 1.5$ centimeters on the upper cervical lip without atypical vessels. C: Preoperative sagittal T2-weighted magnetic resonance imaging revealed no endocervical tumor extension. 
imaging revealed no gross mass invasions in the endocervix (Figure 1B). The preoperative serum level of squamous cell carcinoma antigen (SCC-Ag) was 3.6 nanograms per milliliters $(\mathrm{mL})$. We counseled the patient to undergo minimally invasive surgery (MIS), with the choices of robotic surgery and laparoscopic surgery. In consideration of fewer surgical incisions and less postoperative pain, the decision was made to perform a SPART with pelvic lymphadenectomy due to the desire of this patient with stage IB1 cervical cancer to maintain her fertility if possible.

The SPA-RT procedure was performed using an OCTO-Port ${ }^{\mathrm{TM}}$ (dalimSurgNET Corp., Seoul, South Korea) to enter the abdominal cavity by open technique at the umbilicus. An ENSEAL ${ }^{\circledR}$ G2 curved tissue sealers device [Ethicon Endo-Surgery, Cincinnati, Ohio, the United States of America (USA)] was used as the energy source. A systemic pelvic lymphadenectomy was performed bilaterally including the deep inguinal, external iliac, internal iliac and obturator lymph nodes, with the specimens separately removed via an Endobag. After that the uterine artery was isolated and dissected from its branch of the hypogastric artery. Then the vesicouterine peritoneum was incised to enter the vesicouterine space and the incision extended laterally and caudally to identify the vesicouterine ligament and the vaginal margin, respectively. The ureter was hung using a vascular loop and pulled laterally to mobilize it down to where the uterine artery crossed over it. The loose tissue between the uterine artery and the ureter was dissected to create a ureteric tunnel, following which the uterine artery was sealed and cut at its original branch (Figures 2A-B). The anterior leaf of the vesicouterine ligament over the ureteric tunnel was dissected downward to where the ureter entered the urinary bladder. Then the paracolpium was dissected separately to push the ureter laterally. Next, a rectovaginal space was formed to identify the posterior part of the vaginal margin. To avoid too much manipulation of the cervix and due to concern about cancerous spillage, we did not use the uterine manipulator. A $\mathrm{KOH}^{\mathrm{TM}}$ Cup (CooperSurgicalTrumbull, Connecticut) with a Hegar dilator no. 13 was used to identify the vaginal margin, after which a colpotomy was created $2 \mathrm{~cm}$ below the cervix by a monopolar energy excision using a Surgiwand (Surgiwand II $5 \mathrm{~mm}$, Covidien, USA). After that, the anterior and posterior vaginal walls were grasped with grasping forceps to avoid tumor spillage. The uterus was then flipped over and extracted through the OCTOPort $^{\mathrm{TM}}$ outside the abdominal cavity, and a circumferential excision made using a scalpel at the isthmic portion of the uterus (Figures 2C-D). The cervix, upper part of the vagina and parametrium were sent for frozen section examination which reported an adenocarcinoma of the cervix with free resection margin (Figures 2E-F). Finally, cervical cerclage was done with a non-absorbable suture using the McDonald technique and uterovaginal anastomosis was performed laparoscopically with Vicryl $^{\circledR} 1-0$. Estimated blood loss was $50 \mathrm{~mL}$ and the operative time was 220 minutes.

The final histopathological results reported a $2.5 x$ $1.5 \times 1.0 \mathrm{~cm}$ adenocarcinoma with a $1.9 \mathrm{~cm}$ length of the vagina tumor-free. The endocervical margin was $1.8 \mathrm{~cm}$ distant from the tumor. There was no cancer involvement in the parametrium, surgical margins or any of the 19 lymph nodes. The patient was discharged 2 days after the operation with a retained Foley catheter during the post-operative period to ensure urinary bladder function, which was removed after 7 days of uneventful voiding. The patient resumed her normal menstrual cycle a month after the operation. A magnetic resonance imaging (MRI) of the pelvis at the third month after the operation showed no evidence of tumor recurrence. Liquid-based cytology and HPV genotyping were also negative during the follow-up period. She became successfully pregnant 


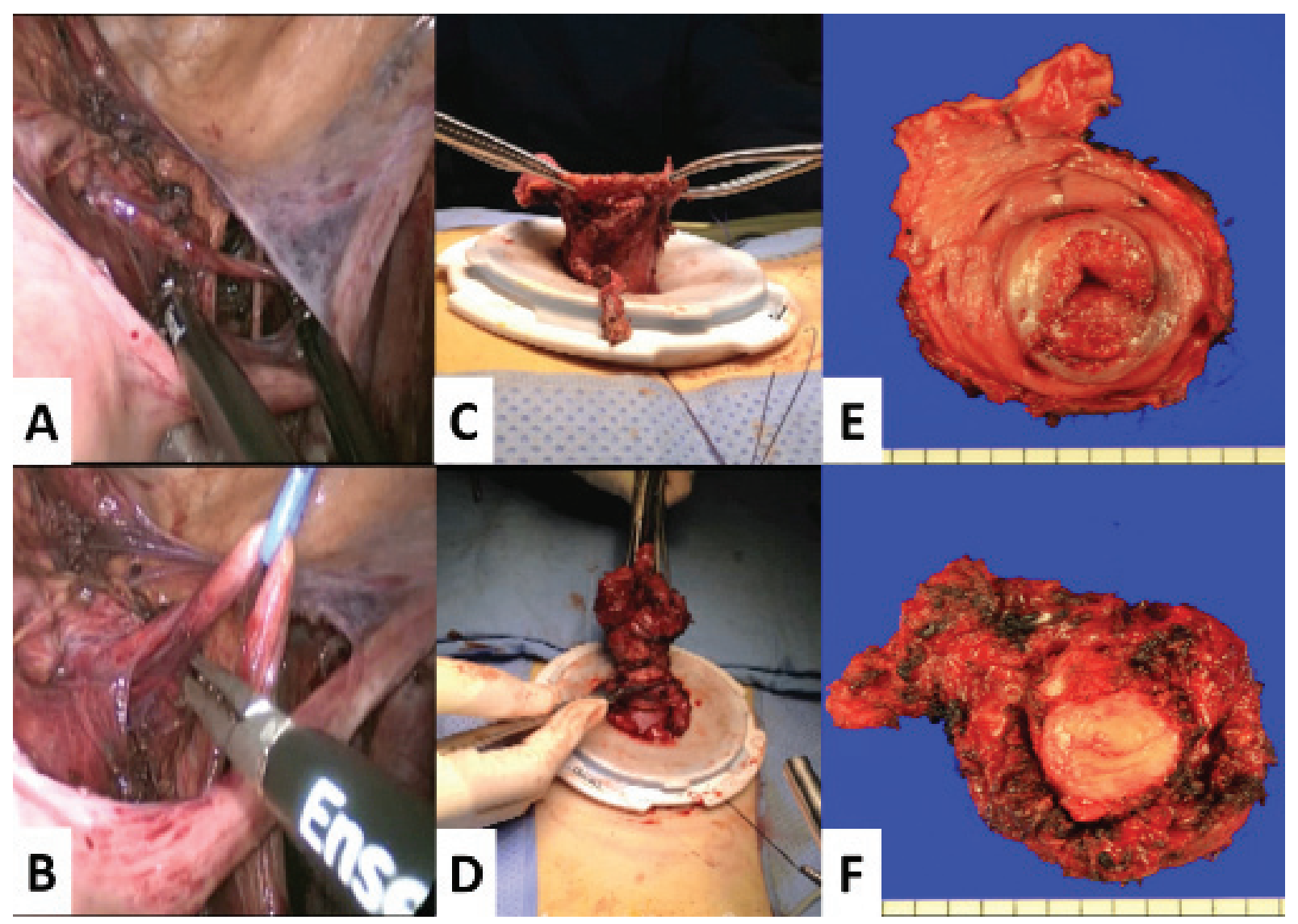

Figure 2 A, B: Demonstrating the ureteric tunnel procedure by using grasping forceps traction at the vascular loop C: The uterus was flipped over and extracted through the surgical port outside the abdominal cavity after the laparoscopic colpotomy. D: The cervix was excised at the isthmic portion of the uterus for an intraoperative frozen section to identify the surgical margins. E: Gross specimen of the cervix and parametrium on the ectocervical part, and F: the endocervical part.

at the fifth month after the operation with no obstetric complications through her pregnancy. She was scheduled for a low transverse cesarean section at 34 weeks of gestation and delivered a viable female infant weighing 1,960 grams with Apgar scores of 8 and 9. Estimated blood loss was $1,000 \mathrm{~mL}$ and there were no post-operative complications. She was followed up every 3 months with cytology, HPV genotype and squamous cell carcinoma antigen testing, and an MRI to check for tumor recurrence every 6 months; as of this writing, 64 months after the operation, all investigations have been negative and she is continuing the surveillance schedule.

\section{Discussion}

Radical trachelectomy is an alternative to radical hysterectomy for women diagnosed with early-stage cervical cancer desiring to preserve their fertility. There are two aspects to consider in performing this procedure. First is the oncological outcome that is usually considered in terms of recurrence and survival rates. Second is the 
obstetric outcome, including both pregnancy and live birth rates. To achieve success in both measures, there are recommended criteria for patients selected for radical trachelectomy including age less than 40 years, strong desire to preserve fertility, common histology (squamous cell carcinoma, adenocarcinoma or adenosquamous carcinoma), early stage (IA1 with lymphovascular space invasion, IA2 or IB1), tumor size less than $2 \mathrm{~cm}$, upper endocervical involvement ruled out by pelvic MRI, no extracervical involvement, and resolution of acute inflammation (4-6 weeks after conization). ${ }^{1}$ Our patient met all of these criteria. Pretreatment serum SCC-Ag level has been reported as a predicting factor for parametrial involvement ${ }^{4}$, nodal metastasis ${ }^{5}$ and detection of recurrence. ${ }^{6}$ This investigation may provide information not only for the patient before undergoing surgery but also for the surgeon to select good candidates for this procedure. Additionally, this tumor marker can be used in the follow-up period. Nevertheless, the serum SCC-Ag may have limited clinical use in the adenocarcinoma cell types, particularly in cases of abnormal preoperative values because high levels of SCC-Ag have been reported in only $25.0 \%$ of adenocarcinoma histologies. ${ }^{7}$

MIS has become the generally preferred surgical approach for many gynecological oncology procedures because of some important benefits over laparotomy. Vieira et al. reported that the MIS radical trachelectomy resulted in less blood loss and shorter hospital stay. ${ }^{1}$ Oncologically, the laparoscopic radical trachelectomy is feasible and safe as shown by recurrence and death rates of $11.0 \%$ and $1.3 \%$, respectively. The reported risk factors for recurrence are tumor size greater than $2 \mathrm{~cm}$ and depth of stromal invasion greater than $50.0 \%{ }^{8}$ Therefore, these factors require assessment when considering this procedure. Concerning histological subtype, there is no difference in the rate of recurrence between adenocarcinoma and squamous cell carcinoma. ${ }^{9}$ Another indicator of the success of laparoscopic radical trachelectomy is the post-operation pregnancy rate, which has been reported at up to $52.0 \%$ after this procedure. ${ }^{10}$ SPA-RT is a challenging surgical approach due to its complexities of both single port access and the radical trachelectomy procedure which require an experienced surgeon with high surgical skills. To our knowledge, this paper presents the first SPA-RT case report in the literature. The procedure statistics show no significant differences from those reported in a previous study on conventional laparoscopic radical trachelectomy in operative time [220 minutes vs. 290 minutes (mean)], estimated blood loss [50 mL vs. $393 \mathrm{~mL}$ (mean)] and length of hospital stay [2 days vs. 9 days (median)], and we had excellent oncologic and obstetric outcomes in a more than 5-year follow-up period..$^{8}$ Nonetheless, according to the results of the laparoscopic approach to cervical cancer trial in 2018 that reported a negative impact on the oncological outcomes in minimally invasive radical hysterectomy ${ }^{11}$, all patients should be informed about the outcome of that trial before undergoing surgery by minimally invasive approach for cervical cancer. Additionally, gynecologic oncologists should establish the optimal indications for performing the procedure and surgical techniques for prevention of tumor spillage to ensure oncologic safety.

\section{Conclusion}

In conclusion, we believe our case reports shows that the SPA-RT procedure is feasible and safe when performed by an experienced surgeon for women diagnosed with early stage cervical cancer wishing to preserve their fertility. Careful patient selection is a cornerstone for achieving optimal outcomes. 


\section{Acknowledgement}

The authors are thankful to the Office of International Affairs (Mr. David Leslie Patterson) for editing the manuscript.

\section{Conflict of interest}

All authors declare no conflicts of interest.

\section{References}

1. Vieira MA, Rendon GJ, Munsell M, Echeverri L, Frumovitz M, Schmeler KM, et al. Radical trachelectomy in earlystage cervical cancer: a comparison of laparotomy and minimally invasive surgery. Gynecol Oncol 2015;138:585-89.

2. Jung YW, Lee M, Yim GW, Lee SH, Paek JH, Kwon HY, et al. A randomized prospective study of single-port and four-port approaches for hysterectomy in terms of postoperative pain. Surg Endosc 2011;25:2462-9.

3. Yim GW, Jung YW, Paek J, Lee SH, Kwon HY, Nam EJ, et al. Transumbilical single-port access versus conventional total laparoscopic hysterectomy: surgical outcomes. Am J Obstet Gynecol 2010;203:26.e1-6.

4. Yamazaki H, Todo $\mathrm{Y}$, Okamoto K, Yamashiro K, Kato $\mathrm{H}$. Pretreatment risk factors for parametrial involvement in FIGO stage IB1 cervical cancer. J Gynecol Oncol 2015;26: 255-61.

5. Takeshima N, Hirai Y, Katase K, Yano K, Yamauchi K, Hasumi $K$. The value of squamous cell carcinoma antigen as a predictor of nodal metastasis in cervical cancer. Gynecol Oncol 1998;68:263-6.

6. Rose PG, Baker S, Fournier L, Nelson BE, Hunter RE. Serum squamous cell carcinoma antigen levels in invasive cervical cancer: prediction of response and recurrence. Am J Obstet Gynecol 1993;168:942-6.

7. Porika M, Vemunoori AK, Tippani R, Mohammad A, Bollam $\mathrm{SR}$, Abbagani S. Squamous cell carcinoma antigen and cancer antigen 125 in Southern Indian cervical cancer patients. Asian Pacific J Cancer Prev 2010;11:1745-7.

8. Park JY, Joo WD, Chang SJ, Kim DY, Kim JH, Kim YM, et al. Long-term outcomes after fertility sparing laparoscopic radical trachelectomy in young women with early-stage cervical cancer: an Asan Gynecologic Cancer Group (AGCG) study. J Surg Oncol 2014;110:252-7.

9. Bentivegna E, Gouy S, Maulard A, Chargari C, Leary A, Morice P. Oncological outcomes after fertility-sparing surgery or cervical cancer: a systematic review. Lancet Oncol 2016;17:e240-53.

10. Ebisawa K, Takano M, Fukuda M, Fujiwara K, Hada T, Ota $Y$, et al. Obstetric outcomes of patients undergoing total laparoscopic radical trachelectomy for early stage cervical cancer. Gynecol Oncol 2013;131:83-6.

11. Ramirez PT, Frumovitz M, Pareja R, Lopez A, Vieira M, Ribeiro $\mathrm{R}$, et al. Minimally invasive versus abdominal radical hysterectomy for cervical cancer. N Engl J Med 2018;379: 1895-904. 